\title{
Dynamic Modeling Method of Cable for Flexible Cable Traction Parallel Mechanism
}

\author{
Shi Chen ${ }^{1}$, Zhibin Wang ${ }^{1}$ \\ ${ }^{1}$ SANJIANGUNIVERSITY, Nanjing, 210000, China
}

Keywords: Cable; Parallel Mechanism; Kinematics; Dynamics; Simulation

\begin{abstract}
Integrated with features of rigidity and flexible cable parallel mechanism, the new configuration of the parallel mechanism based on rigid-flexible combination has been proposed. The existing parallel mechanisms mostly are developed based on the typical Stewart configuration, and the driving components are rigid drivers. However, in recent years, the new flexible cable traction parallel mechanism are completely flexible driver - flexible cable parallel mechanism, which has many advantages of the parallel mechanism, and greatly and simultaneously reduce the mechanism weight for the flexible cable driver instead of rod driver is available to such mechanism, so that the mechanism is characterized with high bearing capacity and low energy consumption etc.
\end{abstract}

\section{Introduction}

Compared with other traditional mechanisms, the parallel mechanism has the characteristics of large rigidity, high accuracy and high dynamic performance, etc, which meets the requirements of modern machines for heavy load, high accuracy and high rigidity of the mechanism. Therefore, it is widely used in machine tools, medical instruments, aerospace, precision positioning devices, industrial robots and other fields.

The parallel mechanism can be defined as the closed loop mechanism in which the moving platform (or end effector) is connected with the fixed platform through at least 2 independent kinematic chains and which two or more degrees of freedom is provided for and is driven in parallel. It is a new mechanism generated with the development of the mechanism theory, and dates back to 1930s.

\section{Introduction to the Configuration and Performance of New Parallel Mechanism}

The new parallel mechanism, i.e. the parallel mechanism based on rigid-flexible combination making full use of advantages of the flexible cable parallel mechanism and proposed on the basis of the typical STEWART parallel mechanism, is researched in the paper. The schematic diagram of the mechanism is shown as Fig.1. The flexible cable is accessible to the mechanism, so as to improve its relevant dynamic characteristics without affecting the kinematic characteristics of the mechanism, so that the parallel mechanism can be better applied in the actual production process.

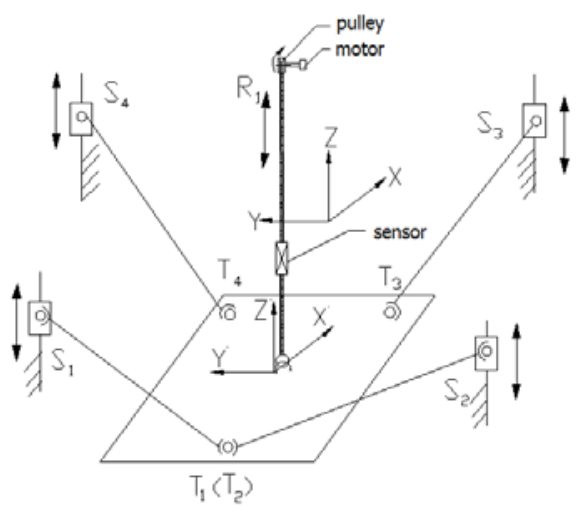

Fig.1. Schematic diagram for new parallel mechanism configuration 
The moving platforms in T1, T2, T3 and T4 are respectively connected with 4 fixed rods with hinges, and ends of the connecting rod are respectively linked with four sliding blocks moving along the rack with hinges in S1, S2, S3 and S4, where the kinematic joint in S3 and S4 is the revolute joint $(\mathrm{R})$ with 1 degree of freedom; that in S1 and S2 is the universal joint (U) with 2 degrees of freedom, and that in T1, T2, T3 and T4 is the ball joint (S). Refer to the fixed form of flexible cable in parallel cable-driven robot, and a flexible cable is accessible to the mechanism. One end of the flexible cable is connected with the moving platform by a thimble, and the thimble of connecting piece is like the universal joint to realize the circumferential motion; the other end is driven by a motor, and fixed on the pulley for connection with the motor. Driven by the main shaft, the pulley is rotated, and controls the elongation and constriction of the flexible cable for synchronous change with the motion of the moving platform.

The mechanism has the motion along the $\mathrm{Y}$ and $\mathrm{Z}$ directions, the rotation around the direction of $\mathrm{X}$ and that around the axis of T3-T4. When the input of four sliding blocks in the mechanism is determined, the moving platform has a definite relative motion.

\section{Kinematics Simulation Analysis}

The parallel mechanism model with four degrees of freedom established under the ADAMS software environment is shown as Fig.2, and the model will be used for simulation analysis on the parallel mechanism. The parallel mechanism consists of moving platform, static platform (frame), fixed rods (4), flexible cable and sliding blocks (4), and the above parts are successively added to the model with the parts library provided in ADAMS/VIEW. The connection constraints among components are described in the Part 2.

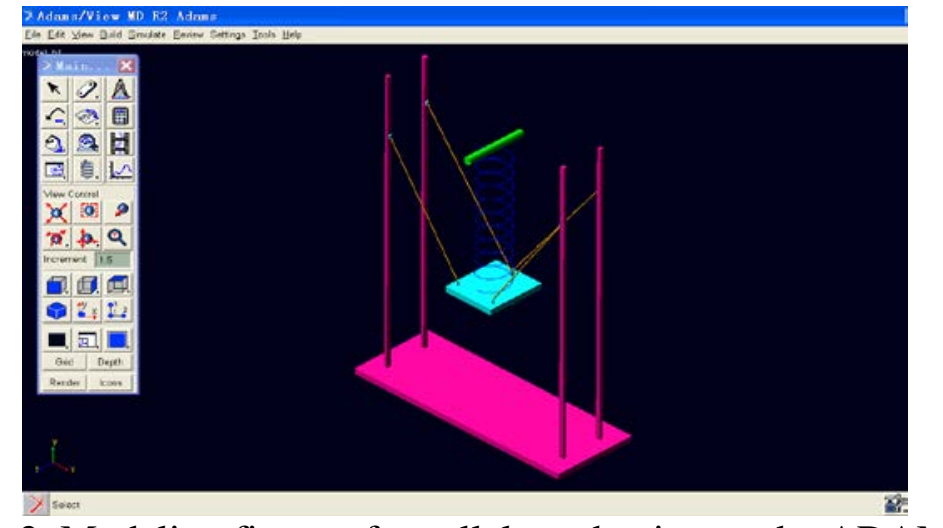

Fig.2. Modeling figure of parallel mechanism under ADAMS

The purpose of kinematic simulation analysis of the mechanism is to examine the relative motion state of each component, check whether the branch hinge has interference, and investigate and evaluate the characteristics of the system speed and acceleration. Change rules of position, speed and acceleration of sliding block in the mechanism under the motion of the moving platform is mainly researched here.

In ADAMS/VIEW, to find out the motion rules of each sliding block when the moving platform moves in accordance with any motion rules, the motion excitation with generic point applies to the moving platform of centroid point, which is shown as the formula (1). The time function is defined with the function generator provided by ADAMS/VIEW, so that the motion of such motion excitation in all directions is constructed and the moving platform has reciprocating motion along $\mathrm{Z}$ axis when rotating around $\mathrm{X}$ axis. The application method is shown in Fig.3 below.

$$
\left\{\begin{array}{l}
\operatorname{TraZ}=5 * \cos (p i * \text { time }) \\
A=25^{\circ} * \cos (p i * \text { time })
\end{array}\right.
$$




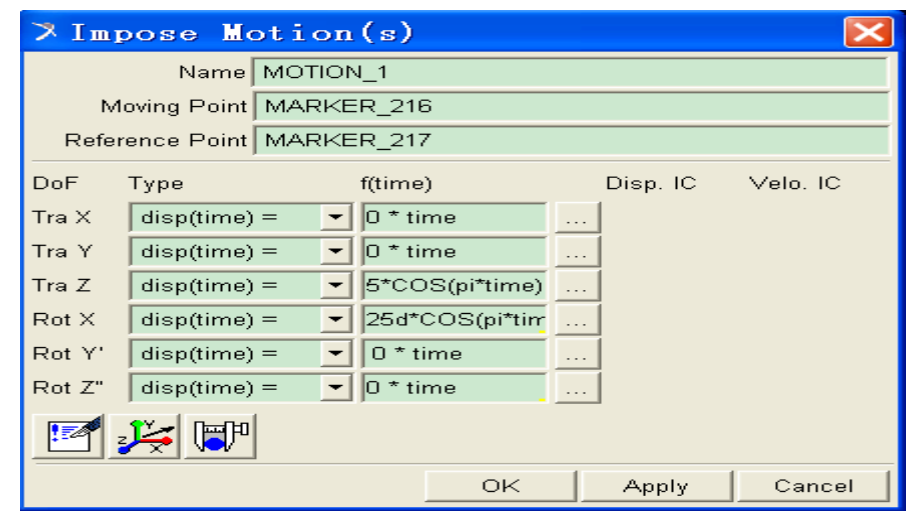

Fig.3. Application of motion of centroid point on moving platform

The object measurement function provided by the application software is used to analyze the kinematics of the model. The simulation of 5S and 100 steps is carried out in ADAMS/VIEW, and the motion excitation defined in the above formula (1) is realized for the platform. Finally, the changing curves of position, speed and acceleration of 4 sliding blocks with time along $\mathrm{Z}$ direction are shown as Fig.4, Fig.5 and Fig.6.

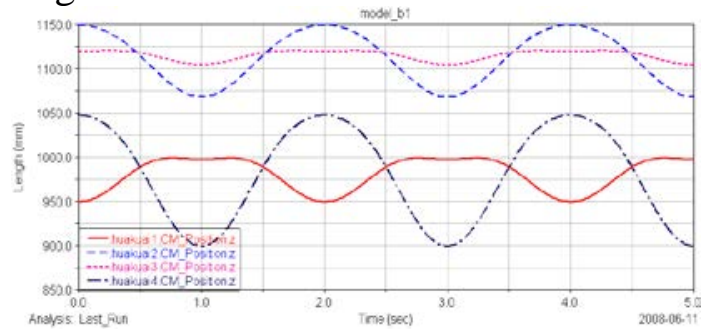

Fig.4. Displacement change of each sliding block along Z direction

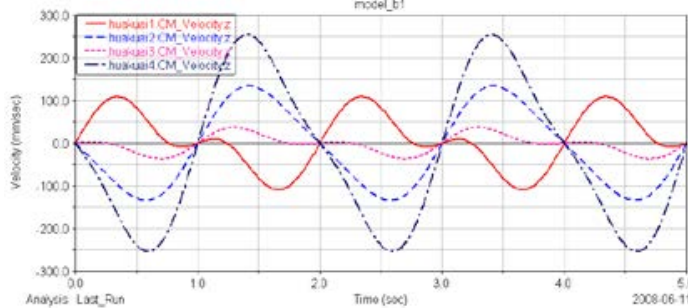

Fig.5. Speed Change of each sliding block along Z direction

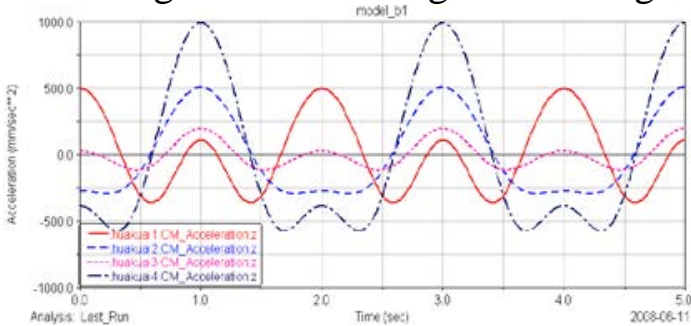

Fig.6. Acceleration change of each sliding block along $\mathrm{Z}$ direction

From the analysis on the above simulation curve, it can be seen that each sliding block has periodic reciprocating motion along $\mathrm{Z}$ axis when the moving platform moves in determined period, and the motion speed, acceleration and displacement changes are stable and not obvious, and will not have impact on the mechanism and meet the basic requirements of mechanism design. The application of flexible cable has no influence on the kinematic performance of the moving platform.

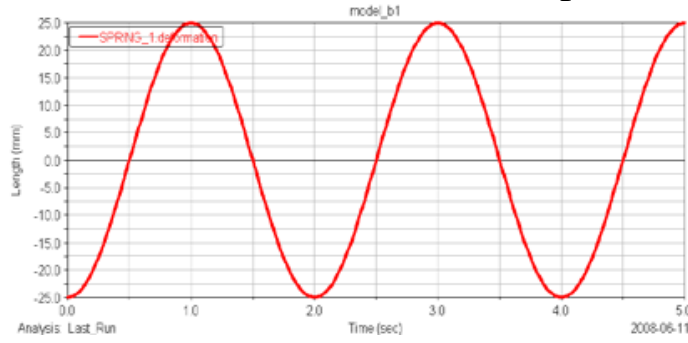

Fig.7. Length change of flexible cable 
With the motion of the mechanism, the flexible cable will produce a certain deformation, and the length of the flexible cable will change smoothly and regularly. The deformation rules of the flexible cable are shown in Fig.7.

\section{Dynamic Simulation Analysis}

\section{Improvement of dynamics analysis of the mechanism}

For the kinematic simulation of the mechanism is carried out in the steady state, the dynamic performance of the mechanism is not considered, and it is less associated with the flexible cable applied. The dynamic simulation analysis on the mechanism is as follows.

First, the any motion excitation is applied to the center point of moving platform, which is shown as Fig.2 below:

$$
\begin{aligned}
& X(t)=200 * \sin (\text { time }), \\
& y(t)=300 * \sin (\text { time }),
\end{aligned}
$$

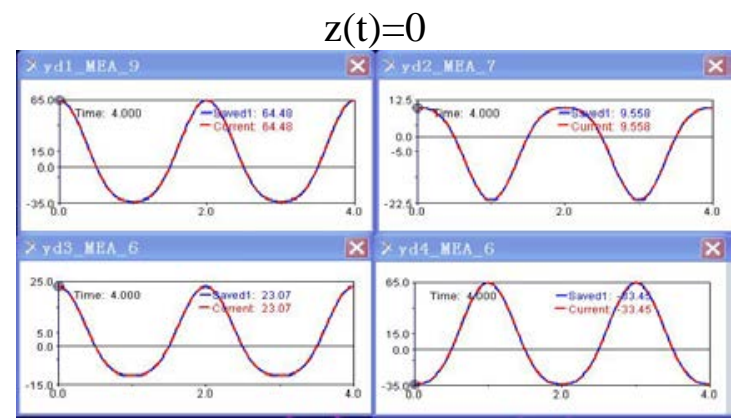

Fig.8. Establishment inspection of displacement measurement of kinematic joint

To obtain the motion trajectory of each sliding block, the displacement measurement (Motion Joint Displacement) is established respectively in sliding joint between 4 sliding blocks and columns, so as to measure the change of position of each block in the process of movement, which is shown as Fig.8.

Then, the dynamic simulation is performed, and the measured position data are generated into 4 spline curves which are defined as s, s1, s2 and s3 in the Adams/Post Processor. Next, remove the excitation motion of cutting-head point of the platform, and the displacement along the column direction is respectively applied to the motion of each sliding block. The displacement curve of sliding block is defined to be same with its spline curve, and the function applied is:

-cubspl(time,0,s,0), -cubspl(time,0,s1,0), -cubspl(time,0,s2,0),-cubspl(time,0,s3,0).

Then, the motion simulation is carried out to realize the desired motion of the moving platform. The $1000 \mathrm{~N}$ force and $1000 \mathrm{~N}^{*} \mathrm{~m}$ moment are applied to the centroid point of the moving platform along the vertical direction, and the force condition of 4 sliding blocks in Y direction can be measured (It is considered that the $\mathrm{Y}$ direction of force is typically the main forced direction in the motion of the mechanism).

Then, under the software analysis and post-processing module Adams/Processor, the changing rules of driving force of each sliding block of the mechanism in the Y direction are output in curve format, which is shown as Fig.9.

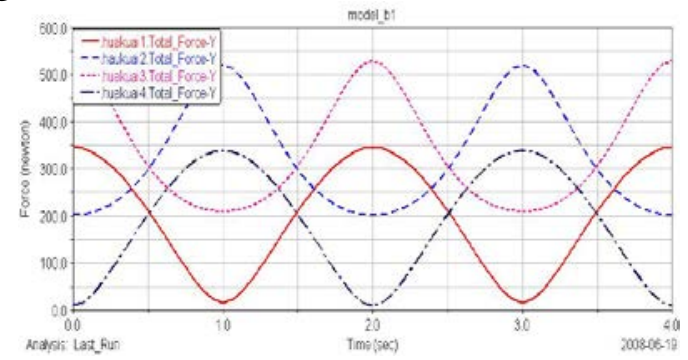

Fig.9. Change diagram of driving force of each sliding block in Y direction

From the above curve, it can be seen that the driving force of each driven sliding block in the mechanism changes continuously and smoothly, and the smooth transition is accessible at the end 
of the curve. Therefore, it has no impact, and the size also conforms to design requirements of the mechanism.

\section{Dynamic analysis of the mechanism without flexible cable}

With the same method and procedure in the above 4.1, the parallel mechanism without the flexible cable is simulated and analyzed in dynamics, and the changing curve of driving force with time is obtained, which is shown as Fig.10.

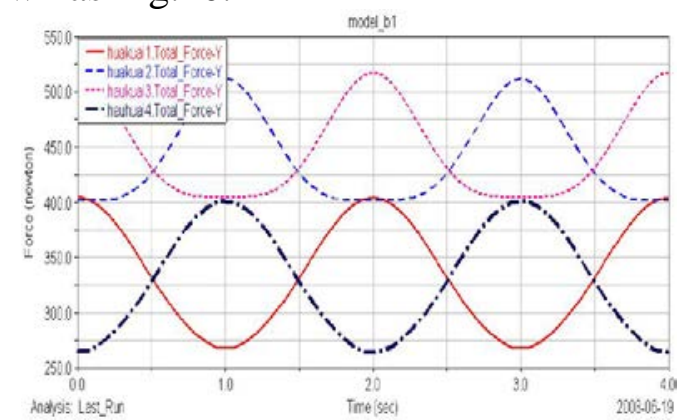

Fig.10. Change diagram of driving force of each sliding block in Y direction

The platform still moves in accordance with the formula (2) as the flexible cable is removed. From the curve, it can be seen that the driving force still changes smoothly, and the starting and ending points of the curve can be smoothly transited without any impact on the mechanism.

\section{Comparative analysis}

With the real-time data comparison, it can be found that the driving force of the mechanism decreases at the initial stage when the flexible cable is applied. It means that in the whole motion process, the initial impact of processing force on the mechanism is reduced. It is shown that the flexible cable applied can improve performance of the mechanism under the premise of no mutation of driving force and influence on the mechanism; accordingly, the initial driving force of the mechanism is reduced and its consumption is decreased.

\section{Conclusion}

With the application of ADAMS software, a virtual prototyping model of new parallel mechanism is established, simulated in terms of kinematics and dynamics, and comparatively analyzed. The flexible cable is applied to the mechanism to improve the accuracy and reduce the vibration of the mechanism. Firstly, the configuration, relevant performance and advantages of the mechanism is systematically introduced, and the virtual prototyping model of parallel mechanism is established with ADAMS simulation software; then, it is simulated and analyzed in the aspects of kinematics and dynamics. Finally, the comparative analysis is made under reference to curve chart of results from the simulation, which confirms that the relevant performance of dynamics has been improved.

\section{References}

[1] Chen Q L, Lin Q. Dynamic Analysis on Flexible Cable-Driven Parallel Mechanism[J]. Advanced Materials Research, 2014, 1006-1007:298-303.

[2] Chen, Qing L, Lin, et al. Dynamic analysis on flexible cable-driven parallel mechanism[D]. Trans Tech Publications Ltd, 2014.

[3] Du J, Agrawal S K. Dynamic Modeling of Cable-Driven Parallel Manipulators With Distributed Mass Flexible Cables[J]. Journal of Vibration \& Acoustics, 2015, 137(2).

[4] Fang L J, Wei Y L, Tao G H. Research on Dynamic Characteristics of Serial-Parallel Combination Joint with Flexible-Cable[C]// Advanced Engineering Forum. 2011:285-290. 